\title{
A Routing Algorithm Based on Zigbee Technology
}

\author{
https://doi.org/10.3991/ijoe.v14i11.9515 \\ Xieji Gang \\ Nanjing Institute Of Industry Technology, Jiangsu, China \\ xiejigang294812@163.com
}

\begin{abstract}
To realize the exploration of A routing algorithm for ZigBee technology, a kind of intelligent parking system based on ZigBee wireless sensor network is designed, and the parking space is managed by online and offline interaction. First of all, the status of parking at home and abroad is studied, and the demand for parking is analyzed. Secondly, the intelligent scheme of parking system is studied, including parking guidance technology and parking space intelligent recommendation technology. The former is based on the A routing algorithm to search the shortest path, and uses Unity for route planning simulation, and the latter is based on collaboration filtering algorithm to discuss the recommendation method of similar parking spaces. The result shows that the prototype of an intelligent parking system based on ZigBee is eventually realized, and the functions of online viewing, online parking reservation, online path planning and parking guidance are realized. As a result, the purpose of managing offline parking spaces through online is achieved, the utilization rate of resources in the existing parking lot is improved and the use rate of parking space is promoted. At last, it effectively alleviates the urban parking chaos and has certain application value.
\end{abstract}

Keywords-ZigBee, intelligent parking, A routing algorithm, Internet of things (IoT)

\section{$1 \quad$ Introduction}

Since the twenty-first Century, the level of the national economy has been greatly improved, the process of urbanization has been promoted, and the population has been increased. As a result, these lead to the emergence of a series of urban problems, among which the most prominent is the urban traffic problem. On the one hand, people's living standards are getting higher and higher, the number of urban private vehicles increase rapidly, and the demand for corresponding traffic facilities is increasing. On the other hand, the construction speed of urban roads and parking lots is relatively slow, causing traffic jam, environmental pollution, parking difficulty and difficulty in traveling. The contradiction between the increasing demand for parking and the number of parking lots provided by the city is becoming more and more prominent, and parking problem has become the bottleneck of the sustainable development of urban traffic.

With the rapid economic development, people's living standards are improved, and the "vehicle family" group is becoming larger and larger. However, when the vehicle brings convenience to people's life, it also causes a considerable number of problems, 
in which the parking disorder and parking difficulty have become the prominent contradiction in the process of urban development. In 2016, several relevant departments of the state jointly issued The Notice on Further Improving the Planning and Construction of Urban Parking Lot and the Policy of Land Use. It encourages social capital to participate in the parking industry, fully digs and utilizes the ground and underground space, and promotes the multi-functional three-dimensional development and compound utilization of the construction land. The intelligent parking service based on multi-functional three-dimensional development and compound utilization will usher in a great opportunity for development. A large number of vehicles have worsened the city's traffic conditions and traffic accidents are occurring frequently. In the meanwhile, a large number of automobile exhaust emissions seriously pollute the quality of the city's air. To solve these problems, the government implements the administrative policy of odd-and-even license plate rule to reduce the pressure and air pollution of the urban traffic system. But this only solves the problem on the surface. In order to solve the problem fundamentally, it is necessary to speed up the construction of urban traffic, combine the new technology and new concept, vehiclery out intelligent management, and rationally plan the urban traffic.

Therefore, it is urgent to develop and design a complete intelligent parking management system to meet the requirements of effective parking management at present. The intelligent parking management system uses highly automated management equipment, combined with computer network, wireless communication technology and Internet technology to manage the parking lot in real time and efficiently. It is necessary to study and establish an intelligent management system of parking lot to realize automatic parking system with advanced technology, control optimization, efficient operation of equipment and centralized management. At the same time, the intelligent parking management system has become the supporting facility for a great number of large and medium-sized comprehensive commercial buildings, schools and residential communities.

\section{$2 \quad$ Literature review}

In the early days, the equipment used in location estimation was compass. In the last century, with the continuous maturity and rapid development of electronic communication technology, many large-scale wireless location systems have been developed. The global positioning system (GPS) is a new generation of space satellite navigation and positioning system developed by the United States in 1970s, mainly used for military purposes. So far, GPS system has gradually entered people's daily life, and GPS navigation mobile phone and vehicle GPS navigator can be seen everywhere, which is convenient for people to know their position whenever and anywhere when they are travelling.

The researchers envisaged the integration of remote wireless mobile communication technology and used the advantages to establish a remote wireless monitoring network with strong expansibility, flexible networking and safe and reliable network. Choe et al. (2017) designed a real-time and dynamic parking guidance system to monitor the 
main parking area, collect the data through the computer controlling the underlying system. The information of the parking lot and the road condition are displayed on the variable electronic road signs, and the rest of the parking space in the surrounding parking lot is updated in real time. The owner of the vehicle chooses the parking lot based on the electronic indicator [1]. Vlahogianni et al. (2016) used the operator's parking guidance and information system, adopted three levels of guidance and display in the district, the intersection and the parking lot to locate the parking lot and the driving direction of the parking vehicle, and update the number of remaining parking space of the parking lot to facilitate the user to choose the parking [2]. Liu et al. (2017) proposed a city intelligent parking system based on Client/Server mode based on Android system. On the basis of location based service, unified management is conducted for the system, which has intelligent parking, intelligent charging, and log records functions [3]. Song et al. (2017) proposed an intelligent parking lot lot navigation scheme based on the ZigBee technology of the Internet of things (IoT). It uses ZigBee and ultrasonic to deal with the vehicle location detection function, and improves the parking efficiency by modifying the shortest path algorithm [4]. Khan et al. (2016) studied wireless remote monitoring based on wireless sensor network (WSN). The WSN composed of ZigBee technology has the characteristics of simple structure, small size, and low cost. It can realize close range wireless connection, which can ensure the real-time and reliability of data transmission [5]. Khan et al. (2016), through the analysis of the new and open wireless interconnection technology ZigBee technology, combined with sensor technology, ZigBee technology and routing protocol, constructed a WSN [6]. Moridi et al. (2018) proposed a CC2431 chip and ZigBee WSN coal mine monitoring system based on received signal strength indication (RSSI) wireless location algorithm. It was promoted by the TI company, which has positioning and tracking engine and has good reliability and real-time performance [7]. Han et al. (2016), aiming at the diversity of the WSN application environment and irregularity effects on the deployment of sensor nodes, a kind of indoor 3D scene reconstruction and 3D ray tracking method is proposed. It can improve the efficiency and accuracy of ZigBee transmission modeling and simulation in indoor environment and can be well used in different indoor environments [8].

To sum up, the above research is mainly aimed at the integration of ZigBee technology, wireless remote monitoring and system data acquisition, but in the selection of methods, it is still deficient. Therefore, based on the above research status, the A routing algorithm based on ZigBee technology is mainly studied, an intelligent parking system based on ZigBee WSN is designed, and the parking space is managed through the online and offline interaction.

\section{$3 \quad$ Method}

\subsection{A routing algorithm}

The A* search algorithm is essentially the best priority search algorithm with constraints, which is used to find the shortest path from a specified point to another in a 
two-dimensional grid. Before using the $\mathrm{A}^{*}$ search algorithm, it is necessary to simplify the problem to a two-dimensional mesh.

If in a two-dimensional grid, $\mathrm{A}$ is the starting point and $\mathrm{B}$ is the end point, if a person wants to go from A to B, how should he go? We do the following operations.

First, start from point A, and use it as a pending point to store in the "startup list". Although there is only one element in the list, there will be more in the future. Your path may pass through the grid it contains (or probably not) and it is just a list of checkerboards.

Second, look for all grids accessible or passable around the starting point and skip the mark of grids that cannot be passed through. Add them to the startup list and save A as the "father grid" of all these grids.

Third, delete the point A from the startup list, add it to a "close list", and close the grids that do not need to be checked again in the list. Next, choose the lowest nearest neighbor grids in the list. Each heuristic search algorithm has an evaluation function. The valuation function of the $A *$ search algorithm is $\mathrm{F}=\mathrm{G}+\mathrm{H}$, and $\mathrm{G}$ moves from the starting point $\mathrm{A}$ to the generated path and moves to the moving cost of the specified grid. H moves from the specified grid to the pre-estimated moving cost of the end point $\mathrm{B}$. Here, the square of the distance from the current lattice to the target lattice is calculated. To continue searching, the grids with the lowest $\mathrm{F}$ value are chosen from the startup list. Then, the following treatment is vehicleried out to the selected grids.

Fourth, find the grids with the lowest $\mathrm{F}$ value, delete it from the startup list, and then add it to the closed list.

Fifth, check all adjacent grids. If they are not in the startup list, add them to the startup list. The new square is used as the selected square grid node. Skip those that are already in or out of the list.

Sixth, if an adjacent grid is already in the startup list, check whether the current path is better. In other words, check whether a lower $\mathrm{G}$ value can be obtained if a new path is used to reach it. If not, don't do anything. If the new $\mathrm{G}$ value is lower, set the parent node of the new grid to the currently selected grid. In this way, keep looking for a lower $\mathrm{F}$ value and looking for the next lattice, until the $\mathrm{B}$ grid is added to the close list. From the grid $\mathrm{B}$, it searches for the parent node until the grid $\mathrm{A}$, which is the shortest path between $\mathrm{A}$ and $\mathrm{B}$. If the $\mathrm{B}$ point is not added to the close list, the $\mathrm{B}$ point is not available.

\subsection{Parking lot detection and control module}

The ca position detection and control module is responsible for the data collection and the hardware control. The data collected by the sensor is the data source of the whole system, and the effective data can drive the system to work properly. The module mainly consists of ultrasonic sensor, Arduino controller, ZigBee module and buzzer, which together make up the vehicle position detection device and hardware control device, and build a typical sensing network. In this sensor network, the sensor is responsible for detecting data, and the ZigBee network is responsible for communicating with the upper layer, and the buzzer is used to respond to the user's operation of booking a vehicle position. 
In practical applications, in extreme cases, a number of mandatory protection measures can be triggered to prevent other users from entering the booked parking space if the user books the parking space. In the parking detection and control module, Arduino is responsible for coordinating each hardware work, and its data interaction is shown in Figure 1.

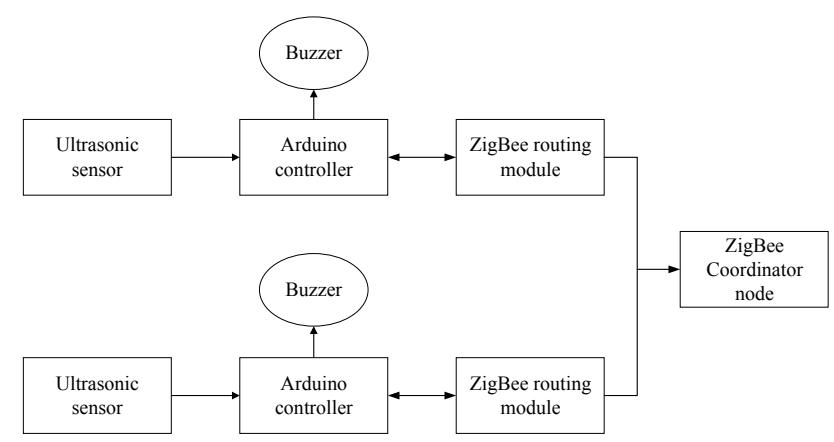

Fig. 1. Parking lot detection and control module structure diagram

Each parking node is controlled by a set of vehicle location detection and control modules. When the status of the vehicle is changed, the sensing data is transmitted to the ZigBee network through the Arduino controller and sent to the host computer serial port. The upper computer submits the data to the upper layer application. In addition, when the control information comes in, the host computer will broadcast the data to each vehicle node through the ZigBee network, and the Arduino controller in the parking lot node reads the control information through serial communication to judge whether it is the target node of the information. If it is not, then discard the information; otherwise, read the control information and control the buzzer working.

\subsection{Data monitoring and processing module of host computer}

In the intelligent parking system, the sensor network ultimately needs to communicate with the Internet. There are two communication schemes at present: one is to install Internet module or WIreless-FIdelity module directly on the hardware system, and the sensor data can be directly submitted to the server. Another is the sensor network to gather data to the host computer, and the host computer is responsible for communicating with the Internet.

For the first scheme, each sensor node has the ability to communicate with the Internet server, and the structure is clear and simple, easy to implement. But a large number of sensor nodes communicating directly with the server will produce a large number of invalid requests and increase the pressure of the server, and the power consumption of the Internet block is large so that it is not suitable for the ow power hardware devices in IoT. This scheme is only suitable for some IoT systems with simple scenes, single functions and fewer nodes. 
For the second scheme, the middle layer, that is, the data processing module of the host computer, is added between the WSN and the Internet. The data processing module of the host computer plays an important role in the system. On the one hand, the host computer and ZigBee central node can read the status information of the vehicle, monitor the running state of the current sensing network, and find and debug the problems in the hardware system of the lower computer in time. On the other hand, the host computer module is designed in accordance with the communication protocol of the IoT (MQTT protocol). It receives the reverse control information from the server and forwards the control information to the ZigBee network through the serial port to control the hardware response. To sum up, the function structure of the host computer data processing module is shown in Figure 2.

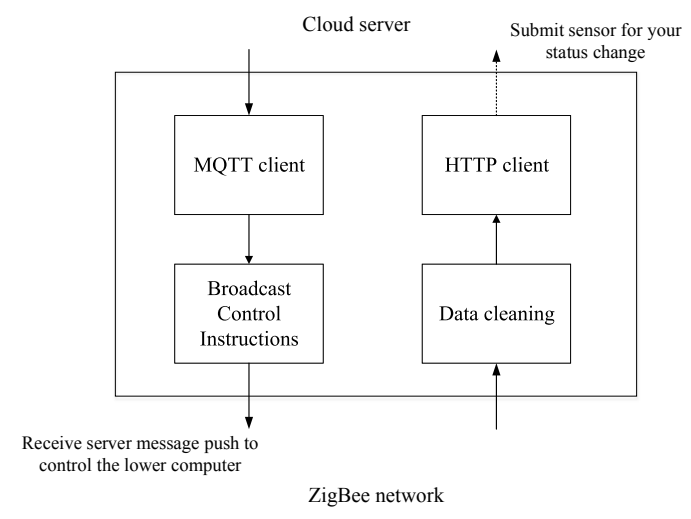

Fig. 2. The function structure of the host computer data processing module

The host computer module monitors the running state of the sensor network, preprocesses the sensor data, submits data to the server, avoids the direct access of the lower machine to the server, reduces the pressure of the server, and receives message push according to the communication protocol of the IoT.

\subsection{Cloud server module}

The IOT system is built on the traditional Internet system. The cloud server must be able to meet high concurrency and high real-time performance because of the requirement of massive data request and real-time control in the IoT. Traditional server development uses reverse proxy, multithreading or server cluster to improve the service performance, but the corresponding technology is also complex and is not suitable for the development of intelligent parking system prototype.

Based on the Node.js platform and MongoDB database, the cloud server programs are developed, and hyper text transfer protocol (HTTP) services and message queuing telemetry transport (MQTT) services are run on the Node.js platform to meet the requirements of different scenarios. The Node.js platform uses JavaScript as a programming language, and the MongoDB database stores data in the format of binary 
serialized document format. It has a good compatibility with the JavaScript language, and the high concurrent query performance is excellent.

In this system, the cloud server needs two-way communication with the host computer and the client. In the process of communication between the cloud server and the host computer, the host computer submits data to the server through the HTTP protocol, and the server pushes the control information to the host computer through the MQTT protocol. In the process of communication between the cloud server and the client, the server responds to the user's request to view the parking space and path planning through the HTTP protocol, and responds to the user's demand for the reservation of the parking lot space through the WebSocket (the MQTT protocol's alternative scheme to the $\mathrm{Web}$ ). The cloud server module is the data storage and distribution center of the intelligent parking system. Its basic structure is shown in Figure 3.

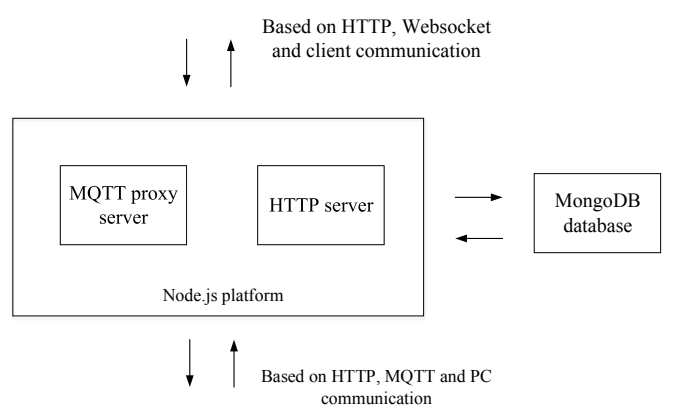

Fig. 3. The basic structure of the cloud server module

\subsection{User client module}

The intelligent parking system will eventually show user client in front of users. In practical applications, in order to facilitate the user's use of intelligent parking system, the client needs to be provided on the mobile device. The Web application based on the browser is designed in consideration of the difficulty of debugging, the difficulty of development and the time cost.

The client provides the basic functions of the intelligent parking system for the user, including the functions of the user registration and login, the statistics of total number of parking spaces, the status of parking space, the book of parking space, and the planning path. The registration and login function is used to save user information, accept user feedback, and restrict users to repeat multiple scheduled parking spaces. After entering the system, users can use parking booking, parking path planning, parking guidance, parking evaluation and other functions. Among them, path planning refers to generate the path from the location of the user to the location of the target parking lot, and the parking guidance means the path from the entrance of a large parking lot to the designated parking space. 


\section{$4 \quad$ Results}

\subsection{Parking guidance based on A routing algorithm}

Extended valuation function: in the parking guidance system, according to the actual situation, the valuation function used in the $\mathrm{A}^{*}$ algorithm is extended. In the general routing problem, the Manhattan distance is used as the valuation function, that is, the Manhattan distance between two points $\left(\mathrm{x}_{1}, \mathrm{y}_{1}\right)$ and $\left(\mathrm{x}_{2}, \mathrm{y}_{2}\right)$ in the plane coordinate system is $\left|\mathrm{x}_{1}-\mathrm{x}_{2}\right|+\left|\mathrm{y}_{1}-\mathrm{y}_{2}\right|$. This method is suitable for planning the path of only horizontal or vertical two ways of walking. In the parking guidance, the car can walk along the slant, that is, in the grid, it can walk along diagonal lines. In this way, the efficiency of the algorithm can be improved. Therefore, the Manhattan distance is extended and its calculation formula is shown below:

$$
\begin{gathered}
\Delta x=\left|x_{1}-x_{2}\right| . \\
\Delta y=\left|y_{1}-y_{2}\right| . \\
h(n)=\min (\Delta x, \Delta y) * \sqrt{2}+|\Delta x, \Delta y| .
\end{gathered}
$$

The extended Manhattan distance represents walking along the shortest diagonal line first and then going straight to the target. It is expressed in a graphic manner, as shown in Figure 4.

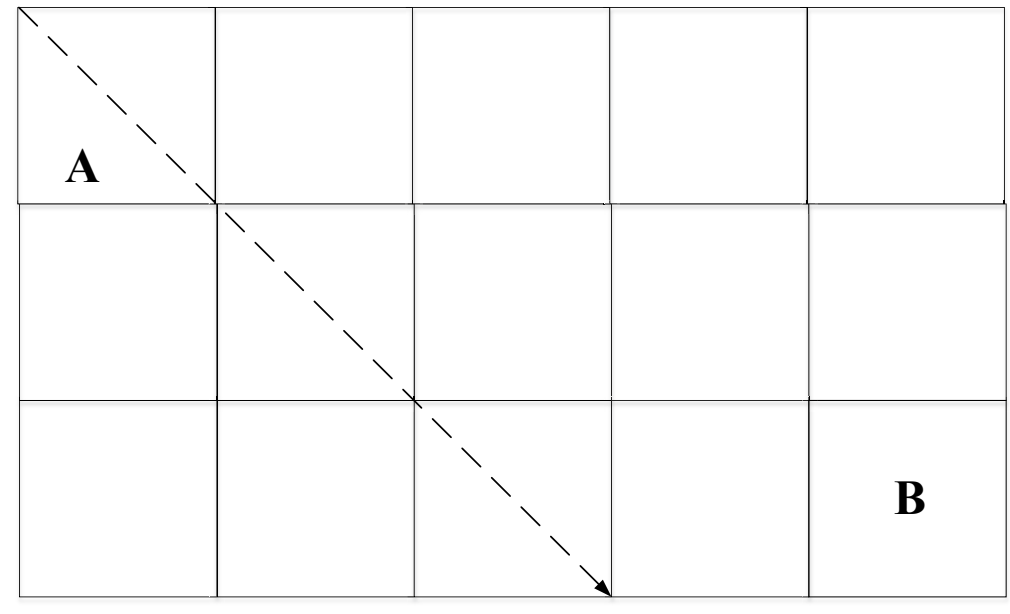

Fig. 4. Extended Manhattan distance calculation method

Execution process of $\mathrm{A}^{*}$ routing algorithm: the most important thing in the implementation of $\mathrm{A}^{*}$ algorithm is to maintain two lists, the open list and the close list. The 
open list stores the nodes to be processed, and the close list stores the nodes that are no longer processed. The specific algorithm flow is shown in Figures 5 and 6.

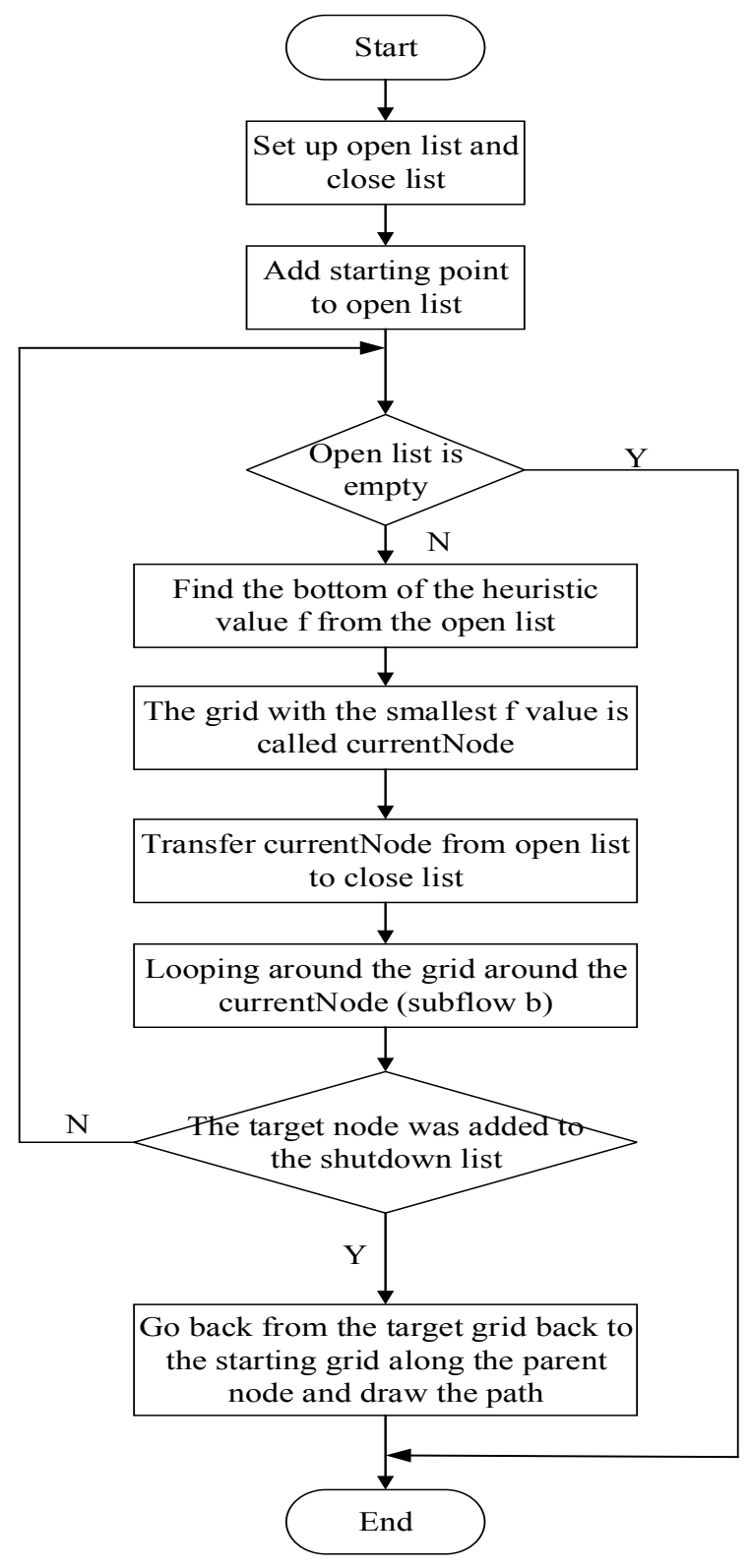

Fig. 5. The process of $A *$ routing algorithm program 


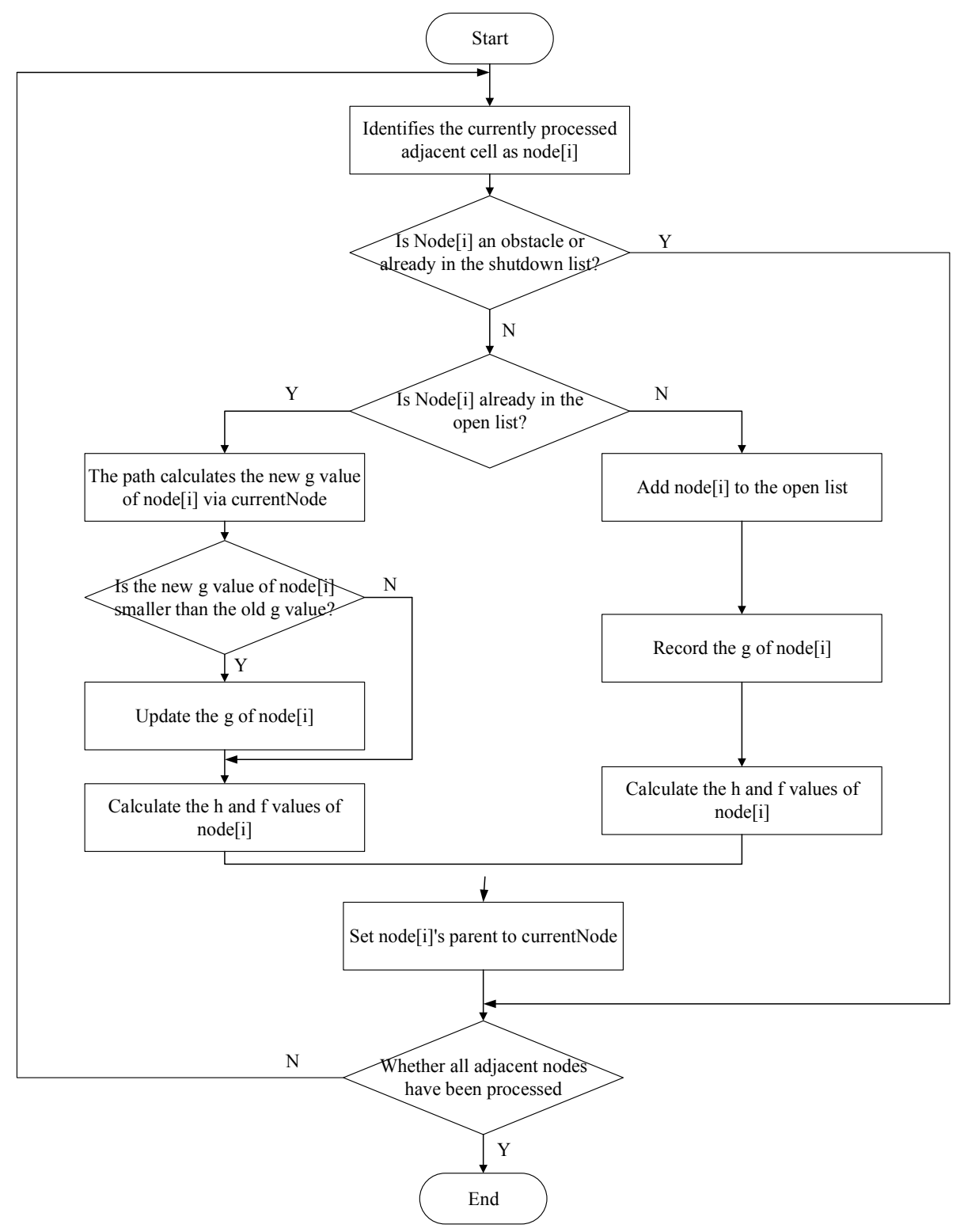

Fig. 6. The sub-process of $A^{*}$ routing algorithm for processing grids

\subsection{Parking lot detection and control function test}

The parking lot detection module is the data source of the system. Through a separate test of the module, the system data can be optimized from the root to ensure the stability of the hardware system.

Analysis of test results: 
First, the data that is read from the serial monitor is the data in a specific format. The format of the data that the data acquisition module of the lower computer sends to the host computer is "SensorId \& cm\&locx \& locy", in which the SensorId value is the node identifier defined in the current Arduino, that is, the number of parking lot. $\mathrm{cm}$ represents the distance detected by the ultrasonic sensor and its unit is centimeter, and locx and locy represent the location information of the current node, respectively.

Second, when the sensor is started, the data detected is not stable, but as time goes on, the data gradually stabilizes and the error gradually decreases.

Third, when obstacle is used in the front of the ultrasonic transmitter, the value read by the serial port becomes smaller immediately, indicating that the module can detect the change of the parking lot. In the intelligent parking system, the ultrasonic sensor is used to detect the change of the parking lot rather than the precise distance value. It is required that the ultrasonic sensor can reflect the change correctly when there is obstacle.

Fourth, when the data acquisition module is tested separately, the data is read directly by the host computer through the universal serial bus to facilitate the debugging, and the data will be transmitted to the host computer through the ZigBee network when the system is run as a whole.

Fifth, when sending a control information through the serial monitor, the buzzer responds immediately, indicating that if the upper application sends the control information in the convention format, the underlying hardware module can make a response. In the experimental environment, the buzzer is used as a response to control information, indicating that the parking lot is marked soft.

The test results show that the parking lot detection and control module can complete the parking status detection and control the hardware response function.

\subsection{Data forward flow test}

The data forward flow refers to the process from the hardware system to the user client interface after a series of system processing of the data collected by the ultrasonic sensor. What finally displayed in front of users is the parking lot status information. This part needs to test the four modules of the system after docking.

Testing procedure: in the process of this part of the test, it is necessary to first assemble and start the lower machine parking lot detection module and the server side program, then start the host computer module which is used as the middle layer. When necessary, the host computer program should be initialized first, and the page will be opened to test the related functions manually. The flow chart of test process is shown in Figure 7. 


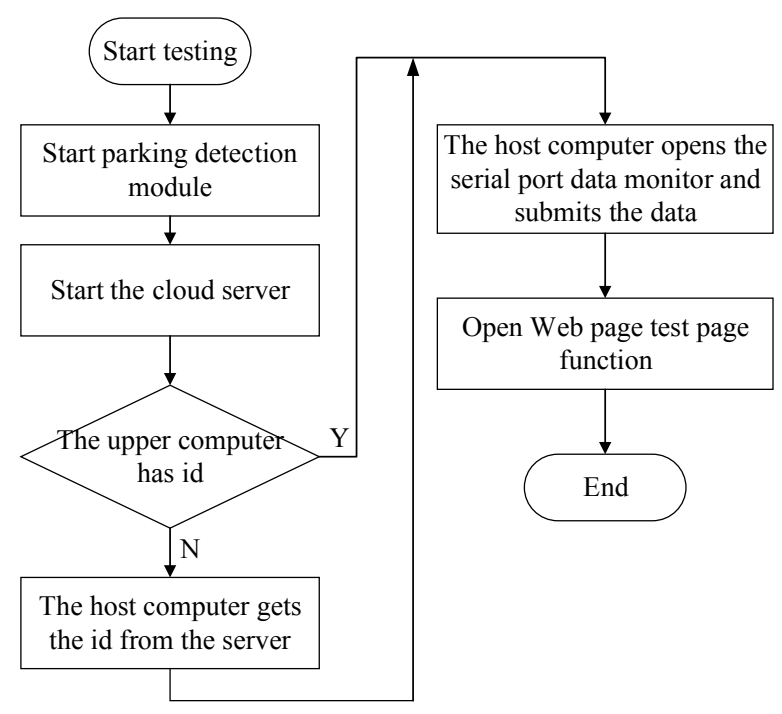

Fig. 7. The flow chart of data forward flow test process

Analysis of test results:

First, the parking lot detection module can effectively monitor the status of the vehicle by ZigBee WSN, and the data are basically stable and correctly displayed in the host computer interface.

Second, the server side runs HTTP services, MQTT services and WebSocket services simultaneously. They are monitored in ports 3000,1883 , and 8081 , and the services run normally.

Third, the identification generation policy is running normally, the initialization data can be stored in the database, and the unique identifier generated by the database will be returned to the host computer.

Fourth, when the distance value measured by the ultrasonic sensor is less than the set threshold, the status of the parking lot becomes occupied, and the change of the parking lot state can also be seen in real time, even if the message is uploaded to the server and the message is pushed to the user client.

Fifth, it can effectively locate the user's current position, plan the path from the user's current position to the specific parking lot, and complete the parking path planning function.

\section{Conclusion}

The demand of the user's parking is analyzed first, the basic function of the intelligent parking system is determined according to the user's demand, and the overall scheme of the system is put forward. The key technology used in it is compared, the technical route of solving the system problem is made, and the overall structure of the system is designed. Under the guidance of the overall structure, each function module 
is realized, including the parking lot detection module, the host computer data processing module, the cloud server module and the Web client module. Finally, the system is analyzed and tested from three aspects, including the data acquisition module of the lower computer (parking lot detection and control module), and the forward flow test of the system data, which verifies the feasibility of the basic function of the whole system prototype. The test and analysis results show that the intelligent parking system based on ZigBee can provide the functions of online parking field search, parking status view, path planning and online parking space reservation for users with parking demand, which can help to solve the problem of parking difficulty.

\section{References}

[1] Choe, H., Gorfman, S., Heidbrink, S. (2017). Multichannel FPGA-Based Data-AcquisitionSystem for Time-Resolved Synchrotron Radiation Experiments. IEEE Transactions on $\mathrm{Nu}-$ clear Science, 64(6): 1320-1326. https://doi.org/10.1109/TNS.2017.2655366

[2] Vlahogianni, E.I., Kepaptsoglou, K., Tsetsos, V. (2016). A real-time parking predic-tion system for smart cities. Journal of Intelligent Transportation Systems, 20(2): 192-204. https://doi.org/10.1080/15472450.2015.1037955

[3] Liu, X., Wang, X., Wright, G. (2017). A State-of-the-Art Review on the Integration of Building Information Modeling (BIM) and Geographic Information System (GIS). ISPRS International Journal of Geo-Information, 6(2): 53. https://doi.org/10.3390/ijgi6020053

[4] Song, Y., Lin, J., Tang, M. (2017). An Internet of Energy Things Based on Wireless LPWAN. Engineering, 3(4): 460-466. https://doi.org/10.1016/J.ENG.2017.04.011

[5] Khan, I., Belqasmi, F., Glitho, R. (2016). Wireless sensor network virtualization: A survey. IEEE Communications Surveys \& Tutorials, 18(1): 553-576. https://doi.org/10.1109/ COMST.2015.2412971

[6] Khan, A.A., Rehmani, M.H., Reisslein, M. (2016). Cognitive radio for smart grids: Survey of architectures, spectrum sensing mechanisms, and networking protocols. IEEE Communications Surveys \& Tutorials, 18(1): 860-898. https://doi.org/10.1109/COMST.2015. 2481722

[7] Moridi, M.A., Kawamura, Y., Sharifzadeh, M. (2018). Performance analysis of ZigBee network topologies for underground space monitoring and communication systems. Tunnelling and Underground Space Technology, 71: 201-209. https://doi.org/10.1016/j.tust.2017 .08 .018

[8] Han, G., Jiang, J., Zhang, C. (2016). A survey on mobile anchor node assisted localiza-tion in wireless sensor networks. IEEE Communications Surveys \& Tutorials, 18(3): 2220-2243. https://doi.org/10.1109/COMST.2016.2544751

\section{$7 \quad$ Author}

Xieji Gang works as Nanjing institute of industry technology, Jiangsu, China.

Article submitted 09 September 2018. Resubmitted 13 October 2018. Final acceptance 17 October 2018. Final version published as submitted by the authors. 